\title{
The Role of Community and Groupware in Geocache Creation and Maintenance
}

\author{
Carman Neustaedter \\ Kodak Research Labs \\ 1999 Lake Avenue \\ Rochester, NY, USA \\ carman.neustaedter@kodak.com
}

\author{
Anthony Tang \\ University of British Columbia \\ 2329 West Mall \\ Vancouver, BC, Canada \\ tonyt@ece.ubc.ca
}

\author{
Tejinder Judge \\ Virginia Tech \\ 2202 Kraft Drive \\ Blacksburg, VA, USA \\ tkjudge@vt.edu
}

\begin{abstract}
Applications that provide location-based experiences are an increasingly viable design space given the proliferation of GPS-enabled mobile devices. However, these applications are in their infancy, and we do not yet know what design factors will contribute to their success. For this reason, we have studied the well-established location-based experience of geocaching. We report on the results of a survey of geocachers along with observations from our own in-depth geocaching activities. Our findings illustrate that geocaching permits users to create a range of experiences for others within a permeable yet restricted culture of norms. Once created, geocaches are maintained by the community of geocachers through a well-designed groupware system. Here maintenance acts can be performed "in the small," given their lightweight and well-defined nature, and become less about maintenance and more about personal participation. These findings provide insight into how community and groupware can be leveraged to support applications for location-based experiences.
\end{abstract}

\section{Author Keywords}

Geocaching, location-based experiences, Global Positioning System (GPS).

\section{ACM Classification Keywords}

H5.3. Information interfaces and presentation: Group and Organization Interfaces-Computer-supported cooperative work

\section{General Terms: Design, Human Factors}

\section{INTRODUCTION}

Many hand-held devices now contain sensors that can pinpoint the location of a user to within a few meters of their real world position. The commoditization of this technology enables the design of location-aware applications and the potential creation of rich location-

Permission to make digital or hard copies of all or part of this work for personal or classroom use is granted without fee provided that copies are not made or distributed for profit or commercial advantage and that copies bear this notice and the full citation on the first page. To copy otherwise, or republish, to post on servers or to redistribute to lists, requires prior specific permission and/or a fee.

CHI 2010, April 10-15, 2010, Atlanta, Georgia, USA.

Copyright 2010 ACM 978-1-60558-929-9/10/04...\$10.00. based experiences. We define location-based experiences as activities that are augmented, prompted, or made possible by content or information that is linked to particular geographic locations.

Systems that deliver location-based experiences are fairly varied in terms of functionality. For example, Darkslide automatically downloads photos previously taken near the user's current location to the user's mobile device [7]. Loopt and BrightKite show users which of their friends are currently located nearby $[3,13]$. O'Hara et al. [17] describe a zoo-based research prototype where children receive more information about an exhibit when they scan a barcode using a mobile device. Many pervasive games often rely on their designers to create elaborate experiences that are tied to real-world locations as the game occurs $[1,2]$. Locationbased experiences are also useful for fulfilling domestic needs. With Place Mail, "users create personal reminder messages, and the system delivers them at user-specified places on their cell phone" [14]. These can further be shared with other users utilizing Sharescape, which visualizes community-created reminders $[15,19]$.

An inspection of this design space suggests that locationbased experiences are made possible by both locationsensing, and perhaps more importantly, the content delivered to users. Given the importance of this content, it serves to study the manner in which it is created and maintained over time. How can systems be designed so that the novelty of the location-based experience does not wear thin (i.e., so that content is fresh, interesting, and useful)? Similarly, how can we ensure that the content does not require constant vigilance and maintenance? Automatically generated content is typically non-rich (e.g., locations of one's contact list) $[3,7,13]$ or can quickly become stale. Many research prototypes rely on the designers or administrators to generate content $[1,2,17]$, which can place a burden on them to orchestrate and ensure the maintenance of the experience. A third approach is to rely on users to either actively or passively generate content for other users to consume $[14,15]$. Such an approach is risky, since it is contingent on users creating and maintaining that content. If users fail to continue to do this, the experiences become nonexistent or stale. 

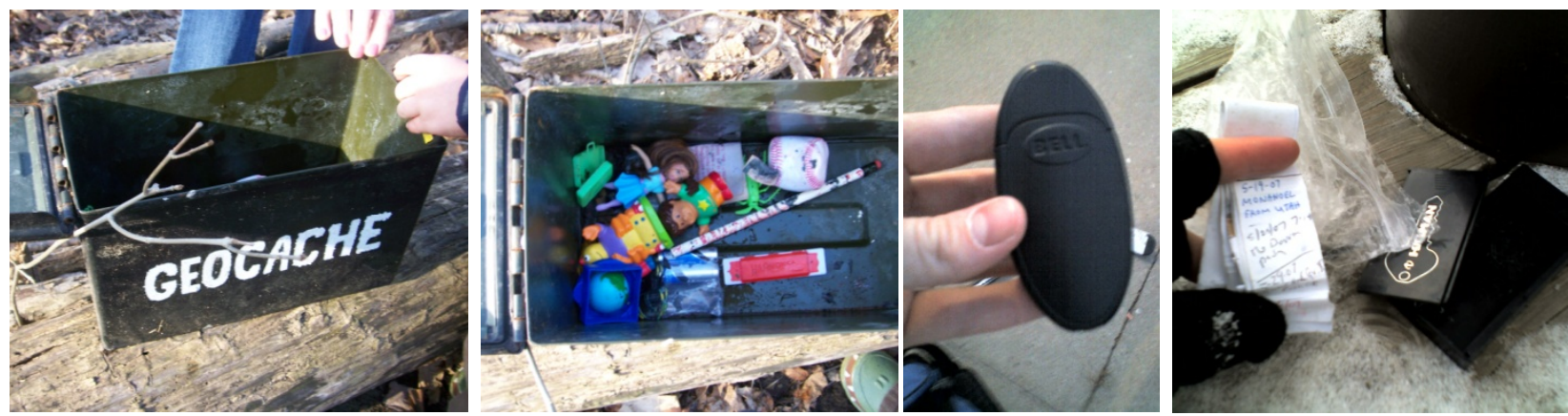

Figure 1. Traditional Caches: ammo can, tradable items, micro cache, and micro cache log book (left to right).

Yet, it is this latter approach-relying on users to both generate and maintain content-that is the cornerstone of geocaching, a location-based activity that has enjoyed considerable success and growth for over a decade. The first geocache was placed in 2000 and today there are over 955,000 geocaches hidden worldwide [8]. To help build our understanding of how geocaching employs this approach, we have studied the creation and maintenance practices of geocachers. This allows us to learn how users construct the experiences, why they find it enjoyable, and what mechanisms support this activity as a whole. This sheds light on how location-based experiences might benefit from similar content-generation and maintenance mechanisms.

Prior work on geocaching by O'Hara [16] identifies the motivations of geocachers for participating in the activity and also describes many of their practices. This identifies the notion of community and shows that geocaching is not just an individual act; people contribute to the activity via online interaction when not out caching. Kelley [10] also describes issues of trust and community in her book on geocaching. Our work builds on this existing literature by taking a deeper look at the manner in which geocaches are created and maintained. Our findings show that geocaching maintains its "freshness" due to the flexibility of the geocache medium: users can design and deploy creations as simple or as rich as they desire. We show that the website that underlies the geocaching community is an effective groupware system that encourages an implicit type of cooperation that in turn has engendered a particular culture of practices and customs. This community helps to maintain a level of consistency in the experiences that others have. In this respect, our results highlight the critical role that community, as mediated by the online website, plays in the creation and maintenance of location-based experiences.

\section{GEOCACHING}

Geocaching is a GPS-enabled treasure hunt. Geocachers (or cachers) hide a geocache (or cache) and then record the GPS coordinates of that location along with a description of the cache onto a common website, the most widely used being geocaching.com [8]. Each cache has its own associated page, where cache creators label the cache with terrain, difficulty, and size ratings from 1 to 5. Cache types vary, but the most commonly placed is a Traditional Cache containing, in the least, a log book (to write one's name, date, etc., when finding it), and sometimes tradable "SWAG" items or small toys (Figure 1, left). Other cache types include Puzzle caches, where a puzzle must be solved to find the location, and Multi Caches, containing multiple waypoints (GPS coordinates) that must be found to reach a final destination. Containers typically range from large ammunition boxes (ammos) to small containers called "micros" created from items such as film canisters or magnetic key holders (Figure 1, right). Geocaches were originally hidden in parks and wooded areas [10], but can now also be found in urban centers.

When seeking out geocaches, users generally search the geocaching website for caches in an area of interest, view specific caches' web pages, and find the caches based on the associated GPS coordinates. Many people download details for a large set of caches onto their GPS device for serendipitous hunting later [16]. Caching is meant to be a secret activity and cachers generally try to be stealthy when hunting to avoid "muggles" (non-cachers) from finding out what they are doing. Caches that have gone missing because of non-cachers are referred to as being "muggled."

Once a cache outing is complete, cachers post logs of their experiences to the geocache web page. Logs can be flagged with attributes, such as found it, did not find it (DNF), or needs maintenance. As we shall see, these simple and largely transient communication mechanisms play a crucial role in helping to maintain individual caches and, collectively, the entire geocaching activity.

\section{RELATED WORK}

Most literature on geocaching is journalistic and does not report in depth about the activity [16]. Forestry researchers, Chavez et al. [5], provided the first research study of geocaching practices with a survey of 133 geocachers in the Minnesota region of the US. They found that people geocached to enjoy the scenery/nature, get exercise, experience new and different things, test their skills and abilities, participate in recreational activities, and learn about natural history. A second article by Chavez et al. [6] articulates the need for management strategies to permit geocachers to continue their activity in forests and parks while still preserving the environment. 
Kelley's book [10] provides the first qualitative look at geocaching practices. Through rich photographic and textual accounts, she illustrates the remarkable similarities in geocaches across the US (e.g., similar containers, terrain, and environment for hides). Kelley goes on to describe geocaching as a means to explore what "community" might mean in the future where humans become augmented with location-smart technologies (e.g., people carrying GPSenabled mobile devices). In this community, she says the key component is thin trust; that is, trust between anonymous people rather than close friends or family [18]. The logs that cachers create of their activities form a reputation system that further enhances this trust. Kelley's account of geocaching is valuable for it sets the foundation for the way we think about community in geocaching. However, it does not provide the methodological rigor needed to understand the details of this community.

To date, O'Hara's [16] study of 14 geocachers in the UK remains the most comprehensive qualitative study of geocaching practices. O'Hara's emphasis is on identifying the motivations that geocachers have for participating in the activity. Here we learn that key motivations include: giving purpose to walks and/or exercise, exploring places, improving online profiles and statistics, feeling challenged, or competing with others. Within these, O'Hara draws out two major implications for location-based experiences. First, people like to intersperse their geocaching activities amidst everyday life activities, such as work, school, or shopping. That is, geocaching is not always an activity in and of itself; it is often part of a larger series of intertwined and ongoing events. Second, geocaching is unique as a system delivering location-based experiences because it draws focus away from the technology itself (i.e., the GPS device) and instead brings it to the real-world location.

O'Hara also shows that community exists in geocaching in several ways. First, caching is not just an activity where one goes and finds caches. A portion of the experience occurs online, where cachers log their geocaching activities and read the profiles of others. Second, we see evidence of community in the small where people will cache in small groups and thus turn the activity into a social occasion. For example, parents might cache with children, or a group of friends might cache together. Third, trust and collective responsibility become important for moveable items known as Travel Bugs (TBs). These are items tagged with a special travel mission. For example, a travel bug placed in a cache in New York City might have the goal of reaching Los Angeles. Cachers then move the travel bug between caches to fulfill this mission. Lastly, O'Hara shows that geocaching is not just a consumption activity. Many cachers spend a portion of their time creating caches for others. In this way, the community is self-sustaining. This last finding confirms research by Salovaara et al. [20] on playmakers in gaming environments.

Our research builds on the concepts of community brought out by the work of Kelley [10] and O'Hara [16]. We focus specifically on the creation and maintenance activities of geocachers to further identify the role of community in the large. Our account illustrates the pivotal role of the community in enabling and supporting geocaching through its central website, which is in turn an artifact-centric groupware system.

\section{METHODOLOGY}

We studied geocaching using two main methods: firsthand experiences and an online survey. The former provided us with a detailed understanding of geocaching and helped us formulate, understand, and interpret the latter.

\section{Geocaching Participation}

We participated in geocaching for a period of ten months. This involved the first author finding 250 geocaches and hiding 10, the second author finding 50 geocaches and hiding 1 , and the third author finding 15 and hiding 1 . This set of experiences is significant for it provides us with firsthand accounts of the activity from the perspective of beginner, intermediate, and advanced cachers. This gives us an understanding of: the range of geocaches available, our own routines for hiding/finding caches, and the strategies employed by others for hiding/finding. In addition, it supplied us with many opportunities for interacting with other geocachers through the geocaching web page logs and messaging system. The disparity between number of finds and hides is consistent with typical geocaching behaviors as reported in our upcoming results and also by O'Hara [16].

\section{Online Survey}

We also created and deployed an online survey that contained sections focused on: evolutions of practices, tools and devices, online interactions, and finding and creation experiences. The survey was created four months into our 10-month participation period. Our firsthand knowledge of the geocaching community helped us understand what would be interesting focal points of study. This also allowed us to: 1) construct questions that would target the types of things we were seeing to gain more information about them from a wider audience, and 2) construct questions that would see if alternative practices were occurring that we were not noticing. Nearly all questions were open-ended because we sought to explore various topics with potentially unknown responses rather than confirm existing theories. For example, sample questions included: "Describe your favorite geocache creation" and "What activities did you engage in to plan the hide?" We pilot tested the survey with several beginner, intermediate, and expert geocachers (some with over 1000 finds) and revised based on their feedback. The survey was then deployed online during months 5 to 8 of our participation and we recruited respondents through two means.

1. Snowball Sampling. We used snowball sampling and forwarded a link to the survey to colleagues and friends who would then forward it on to others they knew. We also posted a link to the survey multiple times on the popular micro-blog site, Twitter. These posts could then be retweeted (forwarded) throughout people's network of 
followers. The survey link remained available for roughly four months, and we received 41 responses of which 29 individuals completed the entire survey.

2. Forums. We contacted 23 regional online geocaching forums throughout the US and Canada and asked permission to place a link to our survey on each forum. We received positive responses from eight forums and posted the survey there. We also contacted the main geocaching forum available at [8]; however, due to forum regulations, we were unable to post our survey to this potentially worldwide audience. Survey links remained available between one and two months, depending on the forum and when they responded to our request to post the survey. Across all forums, we received a total of 206 responses of which 156 individuals completed the entire survey.

We analyzed survey answers during months 8 to 10 of our participation. Our continued participation during this time helped us look for negative cases that might contradict the results we were finding in. None were found. Analysis was performed using open coding [21]. For each survey question, we assigned each participant's answer with a label or code. If that type of answer was already given, we assigned a previously generated code. New answers would receive new codes and so on and so forth. We tabulated the number of codes found for each question to understand which findings were most prevalent, as well as which findings were less prevalent yet still important. Our results present representative quotes from the survey answers, though they are representative of larger trends revealed by our analysis.

\section{Participants}

In total, we had 185 completed surveys. A total of 172 respondents disclosed their location: 115 were from the US, 42 from Canada, 11 from mainland Europe, 3 from the UK, and 1 from Australia. Of the 175 respondents who disclosed their gender, 129 were male $(73.7 \%)$ and 46 were female $(26.2 \%)$. Of the 174 who disclosed their age, the median age was 42 years with a range of 13 to 71 years. We also asked about household composition and 175 people responded: 33 were single with no children $(18.9 \%), 43$ were couples with no children $(24.6 \%)$, and 99 were couples or single parents with children $(56.7 \%)$.

We also asked respondents about their geocaching experience. Twenty-two had geocached for less than 6 months $(14.8 \%)$, 40 had geocached for 6 months to 2 years $(26.8 \%), 52$ for $3-5$ years $(34.9 \%)$, and 35 for more than 5 years $(23.4 \%)$. Respondents were also asked approximately how many caches they had found. The median response was 730 with a range of 2 to 7000. A total of 150 respondents $(81 \%)$ had also hidden at least one geocache and, for these creators, the median number hidden was 9 with a range of 1 to 192 . For cache creators, the median ratio of finds to hides was 52 and ranged from 3 to 2000 . This means that in the case of the highest ratio, the individual found about 2000 caches and only hid one. In the case of the lowest ratio, the cacher found 140 and hid 42 . Overall, these numbers show that the majority of our respondents were experienced cachers, although we also had a smaller number of less experienced, beginner cachers.

\section{CACHE CREATION}

Geocaching relies on users to actively create caches (i.e., the "content" of the game). Because of their lightweight but flexible nature, new caches are constantly being added by the user population. We illustrate how users employ both the richness of the real world and the empty canvas of the website to create a range of different caches. At the same time, we show the role of the geocaching community in maintaining cultural "norms." Respondent quotes in our results remain unaltered, although we have added text in square brackets for clarity or to preserve anonymity.

\section{Flexibility of Cache Creation}

Geocaching.com suggests guidelines and standards for the types of caches people can create and roughly where they might be placed. Before being approved for listing on the public site, the web page for each new geocache is reviewed by a volunteer to ensure that the cache does not contravene any (largely common sense) requirements. For example, the cache must be at least 0.1 miles away from other caches and not at military installations or potential terrorist targets. Thus, there are basic rules but the framework is fairly lax, meaning that geocachers can be quite creative. The pairing of a physical object in a realworld location with the ability to add freeform text and images to the associated web page has also led to many meticulously researched and carefully crafted caches. Yet, because of this low barrier to entry, there also exist many geocaches that are hastily hidden in the urban environment. This ensures that geocaches can be quickly and constantly added to the global game. Interestingly, the entire range of geocache types is valued by the community.

Range of Geocaches. We asked respondents who had hidden geocaches to describe their favorite geocache creation to us. Favorite creations were nearly always composed of careful construction and/or meticulous planning. Here people often researched a location carefully to learn about its terrain and/or nearby caches. They would also purchase supplies to make sure a container would match an area or be concealed well. Some even assessed muggle activity in the area. These activities sometimes took weeks or even months to complete.

\footnotetext{
"We usually take 2 or more trips to hide the cache. We want to make sure that it is a good spot and spend time thinking about whether it is a good location or not." - Female, Age 40, Virginia, USA

"[It took] months of retooling and working through the concept, creating themed clue cards, artwork, containers, seeking out appropriate locations." - Male, Age 40, Illinois, USA

"I was excited that no one had hidden a cache at this location, and since it is one of my favourite spots I wanted to make it a good and somewhat difficult hide. I went out to the location to scope out the best hiding spot and marked the coords in my GPS. I asked the owners for permission to hide the cache on their property. I did some research online to come up with a clever name and to get some info for the cache page. I found an
} 
appropriate container to fit in the spot, spraypained [sic] it, and then decided what swag and FTF [First to Find] prize to include. I wanted to release a TB with the cache, but couldn't find an item that fit in with the theme. I submitted the coords to our review to check that they were ok. I then hid the cache and let the reviewer know it was ready to go." Female, Age 34, Canada

What is clear is that many of these creations are deliberately intended to provide other geocachers with an interesting location-based experience. In many cases, such caches will be of personal significance to the hider or a point of interest useful for educating other geocachers about the location [16]. In such cases, the geocache's web page might have considerable prose describing the significance of the area:

"My husband and I create the caches together. He finds the tree we want
to educate people about, he makes the web page for the site, and I usually
do the hiding and prepare the cache."-Female, Age 40, Virginia, USA

Such efforts are appreciated by other geocachers. When respondents were asked about their favorite geocache find, many similarly prefer to find caches that have had extra attention paid to them with rigorous planning:

"There is a cache in CO which was hidden in a bird house which was about 25 feet in the air and there was a cable which let the cache down. Very cool cache because it was up in the air and very creative" - Male, Age 20, Colorado, USA

"One of my favorites was one that had me puzzled for a while. In the end I figured out that you had to get water and pour it into a pipe that would make the cache float to the top. But you had to hold your finger on a hole at the bottom to stop the water from flowing out."-Male, Age 54, Canada

At the other end of the spectrum, many geocaches are fairly straightforward to find or are associated with minimal description on the web page. Individually such caches (socalled "cache and dashes") might be less favored by people because they are easy to find. However, they are still valuable to the community because many cachers are simply interested in increasing their "find count" (i.e., a personal achievement [16]):

"Everyone always says it's not about the numbers. I think to a lot of people it is about the numbers and getting to a certain point. This is a nice and easy cache that I wanted to hide. Now you can add another number to your caches and reach your goal. I will be putting other ones out like this so you can keep getting the numbers." - Excerpt from Cache Description in New York, US

Newcomers. While the geocaching website suggests cachers wait until they have gained some experience in finding caches before placing their first cache, a large number of relatively new cachers become active in the creation of geocaches early on. The website itself places no restrictions on who can create caches, nor how much "experience" one must have before doing so. As a consequence, caches created by newcomers are not all considered "good" to find. For instance, they might be poorly hidden, not be hidden in interesting places, or suffer from other technical problems.

"I followed the suggestions on the website about having experience before placing a cache. I was almost at 200 finds before I put out my first one. I learned early on that it's very annoying to go look for a cache that is not well done or the coordinates are off. I waited until I felt I had something to contribute to the game before jumping in." - Female, Age 55, USA
Nevertheless, the low barrier to entry for cache creation is still useful. After creating even a simple geocache, cachers immediately become a part of the caching community, feeling a degree of responsibility for it - above and beyond simply being a "finder" of geocaches, they are actually active contributors. Placing a cache brings with it the responsibility of maintaining it and thus a longer term commitment to the activity [16].

Preserving and Evolving the Norms of Cache Creation Although there is an official set of rules to how geocaches are created, an implicit set of customs has evolved through user practices, which establishes a sort of lingua franca. These customs help preserve the norms of geocaching by defining the types of caches that are created, their contents, and where they are placed. Thus, even though cache creation is typically quite flexible, as previously described, the evolved customs offer a counterbalancing mechanism for consistency. This ensures that caches are similar enough between locations that they fit the basic practices of the activity and are understandable by cachers finding them. This lets cachers recognize a cache when they find it and know what to look for. This is important as cache containers often look like everyday objects (e.g., film canisters, Tupperware $\AA$, trash).

Learning Customs. Cache customs are typically learned by cachers as they cache. For each unique cache that people find, they build on their understanding of the activity and, in turn, the customs. Even small variations in caches provide additional knowledge. For example, cachers might find a new style of container (e.g., plastic vs. metal), notice a new method for attaching a cache container to an object (e.g., tied to a tree, magnetized to a light post or bench), or discover a new way to protect the contents of the cache (e.g., plastic bags). The learning of cache customs creates a level of "geosense" that cachers can use to help them find caches. The understanding of cache customs is also valuable for cache creation. Cache creators are not required to follow customs, yet we found that they often do. Creators might feel pressure to conform, given that potentially hundreds of people are "watching" what they do (by finding their cache creations) and even logging their experiences. Cache creators might thus realize a better method for doing something than they would have done otherwise.

"[When I placed a cache] I followed typical expectations. I was new to geocaching and didn't want to rock the boat in anyway. "-Female, Age 25, Virginia, USA

"The original coffee can container was wrapped in trash bags since that seemed to be a standard method of protecting cache containers at the time. It seems now that practice isn't followed as much, which is one of the reasons I replaced the container." - Male, Age 36, Canada

Caches are also hidden by people with little experience who might not yet understand the norms of geocaching. Such caches are essentially "policed" by the community at large through the web logs, which we discuss in the next section.

Evolution of Customs. As is the nature with any type of customs, they evolve. Sometimes cachers will go out of 
their way to create something that is different or unique to an area. This might be done to increase one's personal reputation for creating new and interesting hides. It can also be done simply to try to trick cache finders who might be looking for something they are familiar with.

"[It is in] Plain sight (you can see it 100 feet away), log book only, blends in well enough that it usually takes several trips to find... I wanted it to be challenging and unique to the area. And it was and still is." - Male, Age 50, Illinois, USA

These "new" caches might be specific to an area at conception, but, over time, knowledge of them spreads through traveling and finding them, or word of mouth.

"At present, [my favorite creation] is a waterproof match container, sunk into a pond with a strong magnet on the end. Cachers are required to bring magnetic bait to retrieve. It is based on a cache I was told about in the victoria [sic] area." - Male, Age 29, BC, Canada

"Got the idea for the camo [sic] from another cachers hide in Chino Hills. The idea for the location from another hide in a local park." - Male, Age 18, California, USA

Regionalization. We also found evidence that geographic regions develop their own set of customs or "dialect." These differences are revealed when cachers travel to other regions. We asked respondents how many countries, states, or provinces they had cached in and if they noticed any differences in the caches. Respondents ranged in the number of places they had cached from 1 to 12 different countries (median 1, interquartile range 1 to 2 ) and from 1 to 49 different states or provinces across countries (median 6 , interquartile range 3 to 12 ). Thirty-four out of 104 people $(32.6 \%)$ who answered the question responded that no differences existed between regions. Seventy out of 104 people $(67 \%)$ said there were variations, although these were typically only minor differences. Regions might have preferences for the types and sizes of caches placed there (reported by 53 people), e.g., ammo cans vs. micro caches vs. puzzle caches. Preferences might even supersede what type of cache would fit an area best, as shown in this quote:

"I've cached in 7 US states (Illinois, Indiana, Iowa, Wisconsin, Minnesota, Florida, Washington DC) and 5 countries (Iraq, Afghanistan, Jordan, Qatar, Australia). For the most part, most cache hides are the same. The area in Australia where I cached had a lot of puzzle caches. In other countries they were usually traditional or easy multi's. I noticed in some of the other states that there might be a preference for hiding micros where a regular size would be more appropriate." - Male, Age 39, Illinois, USA

In some cases, respondents said the environment caused differences in the locations of hides, the types of containers used including camouflage, or the density of hides (reported by 27 people). For example, if a region has a large amount of wooded area, caches might be more likely to be found in the woods. The following quote shows other differences:

"I have geocached in Arizona and Ohio. In AZ you have to deal with extreme heat, and in Ohio you have to deal with cold and snow! Ohio has more trees, and AZ has more rocks. ... it's like different worlds completely. Also here in AZ you can have more tins, but in Ohio you have more Tupperware becuase [sic] the tins rust there and in $A Z$ the Tupperware gets chewed up by packrats!" - Female, Age 24, Arizona, USA

Weather in different regions can also play a factor in the types of geocaches that are hidden. The following is an excerpt from a cacher from North Carolina, USA where winter weather typically does not feature snow. Recently he visited New York State, where snow in the winter is common, and placed a cache:

"When visiting from NC I was surprised at the few caches that were on
[the college] campus. I wanted to place more but only had a week and
there were only a few spots that could easily be maintained by family who
work there. It was a different mindset, too, to place caches keeping in mind
that there is often SNOW!" - Cache Description in New York, USA

Ten respondents also commented that the caches in some regions are in general more difficult to find than caches in other regions. Similarly, difficulty ratings in one region might be higher than others when comparing the actual difficulty of finding caches (reported by 6). Terrain ratings might also describe caches hidden in very different terrains.

"II've cached in] BC, Alberta, Washington, and Alaska. I found camouflage and interpretation of terrain to be different in Alberta. In BC, a terrain $3+$ usually means climbing a mountain. In Alberta, it means going through thicker bushes. "-Male, Age 29, BC, Canada

When cachers return home from their travels, they bring with them the customs that they learned and this may affect the way they think about geocaching or create new caches.

Thus, geocaching community members create, pass on, and evolve customs that show how to create caches and where to place them. This provides the foundation for new geocache creations. Despite some differences, caches are basically similar enough between regions that cachers are able to understand them and then adapt their own finding practices accordingly. The fact that geocachers observe these differences and can articulate them emphasizes that the differences between regions are understandable and do not greatly affect the experiences. Thus, geocaching is an activity that is easily done in many areas, even when one is away from home. The geocaching customs provide a framework for the way in which cachers think about new caches they want to create. This overarching understanding of geocaching plays a critical role in allowing the community of cachers to actively create and maintain caches for others. We discuss this maintenance next.

\section{GEOCACHE MAINTENANCE}

Because geocaching relies on physical objects in the real world (c.f. location-aware delivery of information [3,13, 16]), it introduces the challenge of actual, physical maintenance of each cache. Cache creators need to ensure that their geocaches have not been stolen, that they are available to be found, that they have not been washed away, etc. To do so requires physically travelling to the cache site, retrieving, and checking the physical cache. Seventy-two respondents said they did this, but for most this was an infrequent activity. People typically try to create caches local to them [16], but it can still be difficult to find the time to monitor their condition. To further exacerbate the problem, cachers will sometimes place geocaches in locations they do not often frequent (e.g., while on vacation) because it is easy to do so. How, then, are the $860,000+$ geocaches listed on the geocaching.com website 


\begin{tabular}{|l|l|l|l|}
\cline { 2 - 4 } \multicolumn{1}{c|}{} & \multicolumn{1}{c|}{ Found It } & \multicolumn{1}{c|}{ Did Not Find (DNF) } & Needs Maintenance \\
\hline Searcher & $\begin{array}{l}\text { Record accomplishment; } \\
\text { Log one's experience }\end{array}$ & $\begin{array}{l}\text { Log one's experience; } \\
\text { Inform creator }\end{array}$ & Inform creator \\
\hline $\begin{array}{l}\text { Proator } \\
\text { Searcher }\end{array}$ & $\begin{array}{l}\text { Knowledge that no } \\
\text { maintenance needed; } \\
\text { Personal satisfaction }\end{array}$ & $\begin{array}{l}\text { Difficulty rating too low; } \\
\text { Notification that cache } \\
\text { might need attending }\end{array}$ & $\begin{array}{l}\text { Notification that cache } \\
\text { needs fixing }\end{array}$ \\
\hline
\end{tabular}

Table 1. The role of logs for searchers, creators, and prospective searchers.
Found It. All respondents reported that they logged their finds online, most often on the same day as finding the caches or within a couple of days. Logs ranged from simple writings of "TFTC" (Thanks For the Cache) to elaborate stories.

"One of the last caches of the day with [cacher name]. A nice walk across the ballfield, but the dew made the incline rather slippery .. we both went down. We found the cache uncovered - we signed the log and covered it better than found." Found It Log from New York, USA maintained on a regular basis? We found that this maintenance involves three primary mechanisms: online logs, in-place maintenance, and adoption.

\section{The Coordinating Role of Logs}

Rather than routinely visit their caches, the vast majority of cache creators rely on the logs that people write about their cache experiences to help maintain them. That is, cache creators generally rely on others to find their caches, log their experiences online, and report any problems. Creators then read these logs and, if problems are reported, will physically fix the cache as needed.

"I mainly pay attention to the online logs. I do not physically go out and check my caches unless someone mentions there is a problem." - Male, Age 29, Illinois, USA

"I monitor them through emails, but not usually by visiting the site. The cachers arround [sic] here are very responsible and would never let a cache fall into disrepair. It's teamwork!!"-Female, Age 30, Virginia, USA

"The "easy" ones I check on every 6 months or so. The real backwoods ones only get checked if thre [sic] is a reported issue. At least 2 of my caches have not been checked on in over 5 years. They seem to be fine from the logs."-Male, Age 42, Nebraska, USA

The geocaching.com website supports various types of logs and, by default, cache owners receive emails each time a cacher writes a log about their cache. This allows them to receive feedback on the status of their cache. Who takes the time to write these logs? As we shall see, the logs serve several purposes, both for those who write them and for those that consume them, functioning as a simple but sophisticated feedback mechanism. These logs form the backbone of the geocaching activity, helping to sustain an informal communication mechanism between cachers as well as the maintenance process for geocaches. We first describe why cachers engage in this logging practice and then how cachers benefit from the logging mechanism.

With respect to each log entry, there are three geocaching users of interest: the searcher (the geocacher who created the log), the creator (the geocacher who created the cache), and prospective searchers (geocachers who are reading the geocache's web page). We describe these users in relation to three types of logs: Found It, Did Not Find, and Needs Maintenance. Table 1 summarizes the various roles that each $\log$ places for the three user types.
"I normally log the evening of the find, or within a few days. Occasionally, I'll get behind, and save the log data in my PDA until I can log. I pride myself on writing an interesting log, and not just "TFTC" or pasting in the same thing for every find in a day. I believe that the cache hider deserves to receive an interesting $\log$ for the effort it took to hide and maintain the cache."-Male, Age 54, Illinois, USA

Found It logs serve two purposes for the searcher (Table 1: Row 1, Column 1). First, they signal accomplishment: the searcher found the geocache and it gets added to their total count. Second, the logs serve as a sort of "journal" of one's adventures - a blog-like description of one's location-based experiences. Within geocaching.com, users can review all of their previous cache finds along with their log posts.

Found It log entries also serve the creator (Table 1: Row 2, Column 1). First, they indicate that the geocache is still present and available to be found. This means that the creator does not necessarily need to check on the cache in person (and as a result, many creators do not). Second, Found It logs indicate that there is still interest in the geocache (i.e., people are still actively searching for it). This can be very rewarding to cache creators as it indicates that their efforts to create the cache are still enjoyed by others. We found that many creators derive satisfaction from reading the $\log$ entries themselves [16]-akin, perhaps, to being notified each time someone enjoys reading one's research paper.

"II] Get email notifications; to gauge continuing interest. My distant caches in Iceland, Norway, and Saudi Arabia help me to keep in touch with the fine fellow cachers who act as local maintainers for me."-Male, Age 64, Virginia, USA

"We like to see who is finding them and their stories as well as how far they travelled to get there." - Male, Manitoba, Canada

Prospective searchers also benefit when Found It logs are posted: they signal a "go ahead" to proceed and find the cache (Table 1: Row 3, Column 1). In some cases, they might even contain hints as to the cache's specific location. Because cache finds are logged relatively quickly, this community feedback is up-to-date and highly reliable. The logs provide additional details about a cache above and beyond its description and rating to let other cachers know the quality of a cache. Most cachers read this information prior to hunting a cache or bring it with them while caching, either downloaded to their mobile device, accessed with an Internet-equipped device, or with paper printouts. Thus, 
Found it logs are akin to positive reviews for the cache and associated cache creator.

Did Not Find (DNF). Geocachers can also post DNF logs to indicate that they could not locate the cache.

"No luck today ... searched for a while and found nothing but mosquitos [sic]. Had to get out of there!!" - DNF log from New York, US

DNFs are indicative of two possible states: the geocache is no longer present, or the geocache is present but the searcher could not locate the cache. Searchers are somewhat more sporadic in posting this type of log: 65 of 180 people $(36 \%)$ said they logged all of their DNFs and 7 said they never did (3.8\%).

"I always post DNF's so that cache owner might get a better idea of trouble with their cache." - Male, Age 30, BC, Canada

The remaining 115 people (63.8\%) said they only logged some of their DNFs. Of these respondents, 84 reported only logging a DNF if they were certain the cache was missing. For example, they had undertaken a concerted effort, revisited the cache site multiple times, others had also marked the cache as a DNF, or they really suspected the cache to have been muggled. "Certainty" that a cache is missing relies on one's experience and self-confidence.

"I will post a dnf UNLESS I believe that the cache is deliberately made to be difficult... and that the hider will get pleasure from me not finding the cache. In that case, I might not log the dnf, but will still add the cache to my "trouble" list and monitor others logs to either (1) get a hint or (2) decide that the cache was missing, and might now be repaired." - Male, Age 54, Illinois, USA

"I only $\log$ a DNF if I put in a decent effort to search for the cache. If I walked to the coordinates, looked around for one minute, then walked away, I don't consider it a thorough enough search to merit a DNF log." Male, Age 29, Illinois, USA

"I only post a DNF if I'm convinced the cache has gone missing. Usually I think I'm just too stupid to find the cache so I don't post a DNF. I watch the cache and if other people find it I'll go back and try again. When I finally log the find I mention how many times I'd been there previously without finding it." - Female, Age 50, BC, Canada

Clearly, searchers are somewhat ambivalent toward posting DNF logs. On the one hand, if the cache is still present, they might embarrass themselves (that is, the creator's cache was too difficult to find). On the other hand, searchers understand that creators (and the geocaching activity as a whole) benefit from DNF logs. Unlike Found It $\operatorname{logs}$, there is no clear reward for posting a DNF $\log$ - it might in fact be considered a blemish on one's recordexcept that it provides an accurate record of one's activities (Table 1: Row 1, Column 2). However, those who report all of their DNFs typically said they did so because of the value it was to the owner rather than to themselves.

Creators find DNF logs to be highly valuable for understanding the status of their cache and whether or not people find it (Table 1: Row 2, Column 2). Knowing that people could not find a cache suggests two possibilities. First, they might have simply had difficulties finding it. This information coupled with viewing a cacher's online profile and seeing how many caches they have found can distinguish true difficulties vs. difficulties as a result of being a newcomer. Owners can then update the difficulty rating if it warrants it. Second, the cache might no longer be there. This is a crucial piece of information as it means the owner must replace the cache if they want it not to be permanently archived. Thus, there exists a tension between cache creators and searchers in terms of the need for DNFs.

"I monitor the emails daily (or more often). I will physically check a cache only if I have a reason to believe there is a problem. My caches are primarily high-traffic... and I expect to see logs on all of them."-Male, Age 54, Illinois

Finally, prospective searchers see DNFs as a signal to avoid searching for the cache-especially in the case of a long string of DNFs (Table 1: Row 3, Column 2). Several DNF logs in succession are generally an assured signal that the cache is missing. On the other hand, DNF logs give the opportunity to spur competition. For example, a prospective searcher might try to "one up" another cacher who previously logged a DNF.

Needs Maintenance. Log entries tagged with "Needs Maintenance" (NM) are intended by the geocaching site [8] to reflect caches that an owner needs to pay immediate attention to and fix. One hundred fifty-four out of 174 people $(88.5 \%)$ said they posted NM logs. Reasons included if the cache container was broken or the log book was wet (117 people), the cache was not there (17), the log book was full (17), or the coordinates were not accurate (3). The following is representative of a typical NM log:

"One of many finds while camping for the weekend in [local park] with [cacher name]. There was about half an inch of water in the cache box bottom and the log book was too saturated to sign." - Needs Maintenance log from New York, US

Of interest is perhaps the in-place maintenance performed by searchers (as opposed to creators). Fifteen respondents said they would first try to fix the cache themselves and would only post an NM log if they were unable to repair it. Fixes included repairing or replacing a container, drying out a logbook, or replacing a logbook altogether.

"I post a NM log when the cache needs some type of repair or replacement that I was not able to do on-site when I visited it. If I can do a repair, or temporary repair, I will not post a NM but I will send the [cache owner] an email." - Male, Age 54, Illinois, USA

"Found the log soaking wet with no baggie to protect it. I took it with me so it could dry out. Will replace it ASAP and post another note when it is in place again." - Cache log, New York, USA

"Occasionally a cacher will replace a log sheet if it's full." - Male, Age 45, Indiana, USA

In these instances, cachers often carry materials with them that are likely to be needed for cache repair, e.g., logbooks, extra containers, plastic bags. This is interesting for it shows that in-place maintenance is often preplanned, thereby signaling a premeditated feeling of responsibility for the geocaching community and associated geocaches.

"We bring a first aid kit, a cache repair kit, some pens, notbooks [sic] and extra batteries. Also, gloves, extra socks, wet naps and ziploc bags." Male, Manitoba, Canada 
In the case of both NM logs and in-place maintenance, searchers perform these acts specifically for maintenance purposes. Even though they generally take little time to perform, people receive little or no reward for them aside from feelings of helping out the community. This further highlights the fact that cachers actively feel a responsibility for providing feedback about cache states and maintaining caches such that others can enjoy them.

Community Verification. As a whole, all three types of logs also actively serve a role for "policing" or verifying caches that have been placed. This is above and beyond what the official approval process is able to do because the volunteer reviewers do not physically visit each cache. Instead, the community members that do actively seek out the caches act as a form of review. Here they can offer opinions on the precise accuracies of the GPS coordinates, the quality of the container and its contents, the difficulty or terrain rating, and the susceptibility of the container and its contents for becoming easily damaged or muggled.

\section{Abandonment and Adoption}

If a cache is flagged as needing maintenance and the owner does not respond after a given period of time, the cache reviewers will archive a cache, which takes it off the public consumption listing. For example, the following is a $\log$ posted to a cache by a reviewer several months after the cache received a Needs Maintenance log. The owner had failed to fix the cache and re-enable it in this time period.

\footnotetext{
"As there's been no cache to find for months, I'm archiving it to keep it from continually showing up in search lists, and to prevent it from blocking other cache placements. If you wish to repair/replace the cache sometime in the future, just contact us (by email), and assuming it meets the guidelines, we'll be happy to unarchive it". - Cache Log by a New York state Reviewer
}

This kind of abandonment is likely to happen to caches that geocachers create outside of their immediate vicinity (e.g., while on vacation), although it can also happen if geocachers become disinterested in the activity after a period of time, or move to a new region. Geocaching.com supplies one mechanism to help avoid this situation: cachers can mark caches for adoption and advertise them for other cachers to take on as their own caches. In some cases, cachers might move the cache to a new location. Adoption was less commonly reported in our survey, but does show the need for caches (and the responsibility for maintaining them) to change hands between cachers.

"I moved from IL back to VA and had 3 caches that I offered up for
adoption prior to leaving the area. I advertised them on the cache itself
and the local geocaching group website [name removed] which I was an
active member of. Interestingly, they were all puzzle/mystery caches and 3
different local individuals volunteered to watch/take them over. The
process was handled via the established [geocaching.com] procedure after
that." - Male, Age 45, Virginia, USA

"Three of my caches have been adopted. Hopefully they are doing their jobs as owners." - Male, Age 37, Illinois, USA

\section{DISCUSSION AND CONCLUSIONS}

Our findings illustrate the viability of relying on users to actively generate (and maintain) content for a system supporting location-based experiences. More specifically, our case study of geocaching provides us with insight into mechanisms that support this system: the geocaching community, its customs, and the website that underlies the practice. These mechanisms show promise for the design of location-based experiences more broadly.

First, we see that geocaching benefits in two ways from the manner in which users are able to create caches. Cachers can create location-based experiences in an easy, lightweight fashion if desired. This can build the community of creators from the onset of participation. It also makes it easy to increase the number of caches available for others to find. Yet cachers are also able to construct more elaborate location-based experiences if desired. This strengthens and enriches the experiences available, and, consequently, the enjoyment within the community. Thus, we see the need for location-based experiences in general to think broadly about both types of creation paradigms. The two will complement the styles of experiences available and provide experiences for individuals depending on their needs.

Second, we found that community members create, pass on, and evolve customs associated with the types of experiences that are available in geocaching. These norms provide a framework for the way in which cachers think about new caches they want to create, either by conforming to norms or evolving them in creative ways. The rules of geocaching are such that they create permeable bounds in which the experience can grow and mature. Yet they also confine the experiences to reasonable bounds so that evolutions of the practice are small or incremental in nature. Thus, regardless of where one is located, the activity is similar enough that it is still understandable and enjoyable. This is valuable for it keeps the geocaching activity consistent yet stops it from growing stagnant, thereby helping to sustain long-term participation across broad geographic regions. Thus, geocaching addresses Reily et al.'s [19] finding that people felt user-contributed content suffered from inconsistencies. In general, we see the need for location-based experiences to include mechanisms that permit the activity to grow and evolve while still maintaining its core attributes.

Third, our results show that the maintenance of geocaches is predominantly held together by the community through the logging mechanism on the geocaching.com website. This community of finders helps maintain caches by providing feedback to cache owners through logs, and, in some instances, actually fixing and updating caches for the owners. The logging system benefits many users of the geocaching.com site simultaneously, although in different ways. Because searchers typically receive some benefit from logging their activities (e.g., as a personal reward mechanism), and they are relatively lightweight and quick to do, the system works as a whole. Geocachers do not need to exert any additional effort to participate in the system; thus, there is no disparity between work and benefit [9]. 
Maintenance is also easy because maintenance tasks are typically well-defined (e.g., find a cache and write a $\log$ ) and they can be done "in the small." The large collection of these small acts is what makes the maintenance mechanisms within geocaching so successful. All of this is underpinned by a layer of thin trust [18]; people take the logs and experiences of others at face value and weigh this against a cacher's experience as found in their online profile. For these reasons, we suggest that location-based experiences more generally consider providing maintenance mechanisms that are lightweight, well-defined, and focus less on maintenance and more on participation.

In some ways, the community practices within geocaching are similar to the manner in which users construct and maintain content in Wikipedia. In both cases, there are people who maintain the overall activity without compensation. In Wikipedia's case, this is the posting and creating of online articles rather than the creation and maintenance of location-based experiences. Wikipedia, like geocaching, is backed by a successful groupware system that allows users to perform maintenance tasks in the small. Here we see that editors and authors tend to come and go very quickly $[4,12]$ and, as articles mature, it might be more valuable to have a larger set of contributors or maintainers [11]. Yet Wikipedia differs in that the act of maintenance is really about just that-maintenance. In geocaching, maintenance tasks are not always about maintenance; a large part of maintenance is a result of other activities, e.g., users sharing their stories and experiences.

We recognize that our work does come with its limitations. Geocaching is a worldwide activity and our coverage is limited mostly to the US and Canada. However, given the remarkable similarity with the activity across various regions within these countries and the ease at which customs are passed on, it is likely that our findings hold for the broader worldwide geocaching community. We also realize that it is not entirely clear what location-based experiences in general would benefit from being designed with similar attributes and mechanisms as geocaching. Understanding this with certainty would require specific investigation of other location-based experiences where geocaching-like mechanisms are put into place. Yet we do believe that the lessons we have uncovered are valuable and can be used as a starting point for other location-based experiences. Here designers should consider the lessons learned from geocaching and understand how they might apply in the specific situational context that their locationbased experiences reside.

\section{ACKNOWLEDGMENTS}

We thank Kenton O'Hara for discussions of his work and possible future directions.

\section{REFERENCES}

1. Benford, S., Crabtree, A., Flintham, M., Drozd, A., Anastasi, R., and Paxton, M. Can You See Me Now? ACM ToCHI, Vol. 13, No. 1, March (2006), 100-133.
2. Benford, S., Crabtree, A., Reeves, S., Flintham, M., Drozd, A., Sheridan, J., and Dix, A. The Frame of the Game: Blurring the Boundary between Fiction and Reality, Proc. CHI 2006, ACM Press (2006), 427-436.

3. BrightKite, http://brightkite.com/

4. Butler, B., Joyce, E., and Pike, J. Don't Look Now, But We've Created a Bureaucracy: The Nature and Roles of Policies and Rules in Wikipedia, Proc. CHI 2008, ACM Press (2008), 1101-1110.

5. Chavez, D.J., Courtright, R., and Schneider, I. Over the River and through the Woods, Parks \& Recreation, 39, 4 (2004), 68-72.

6. Chavez, D.J., Schneider, I., and Powell, T. The Social Psychology of a Technology Driven Outdoor Trend: Geocaching in the USA, Proc. HICSS 2004, ACM Press (2004).

7. Darkslide, http://connectedflow.com/darkslide/

8. Geocaching, http://geocaching.com

9. Grudin, J. Groupware and Social Dynamics: Eight Challenges for Developers, Communications of the ACM, 37, 1, ACM Press (2004), 92-105.

10. Kelley, M.A. Local Treasures: Geocaching across America. Santa Fe: Center for American Places (2006).

11. Kittur, A., and Kraut, R. Harnessing the Wisdom of Crowds in Wikipedia: Quality through Coordination, Proc. CSCW 2008, ACM Press (2008), 37-46.

12. Kuznetsov, S. Motivations of Contributors to Wikipedia, Proc. SIGCAS 2006, 36, 2, ACM Press (2006).

13. Loopt, http://www.loopt.com/

14. Ludford, P., Frankowski, D., Reily, K., Wilms, K., and Terveen, L. Because I Carry My Cell Phone Anyway: Functional Location-Based Reminder Applications, Proc. CHI 2006, ACM Press (2006), 889-898.

15. Ludford, P., Priedhorsky, R, Reily, K., and Terveen, L., Capturing, Sharing, and Using Local Place Information, Proc. CHI 2007, ACM Press (2007), 1235-1244.

16. O’Hara, K. Understanding Geocaching Practices and Motivations, Proc. CHI 2008, ACM Press (2008).

17. O'Hara, K., Kindberg, T., Glancy, M., Baptista, L., Sukumaran, B., Kahana, Gil., and Rowbotham, J. Social Practices in Location-Based Collecting, Proc. CHI 2007, ACM Press (2007).

18. Putnam, R. Bowling Alone,Simon and Schuster (2000).

19. Reily, K., Ludford, P., and Terveen, L. Sharescape: An Interface for Place Annotation, Proc. NordiCHI 2008, ACM Press (2008).

20. Salovaara, A., Johnson, M., Toiskallio, K., Tiitta, S., and Turpeinen, M. Playmakers in Multiplayer Game Communities, Proc. ACE 2005, ACM Press (2005).

21. Strauss, A. and Corbin, J. Basics of Qualitative Research, 2nd Edition. Chapter 8, Open Coding, Sage Publications (1998). 